TRANSACTIONS OF THE

AMERICAN MATHEMATICAL SOCIETY

Volume 349, Number 4, April 1997, Pages 1377-1387

S 0002-9947(97)01801-1

\title{
THERE ARE NO PIECEWISE LINEAR MAPS OF TYPE $2^{\infty}$
}

\author{
VÍCTOR JIMÉNEZ LÓPEZ AND L’UBOMÍR SNOHA
}

\begin{abstract}
The aim of this paper is to show that there are no piecewise linear maps of type $2^{\infty}$. For this purpose we use the fact that any piecewise monotone map of type $2^{\infty}$ has an infinite $\omega$-limit set which is a subset of a doubling period solenoid. Then we prove that piecewise linear maps cannot have any doubling period solenoids.
\end{abstract}

\section{INTRODUCTION AND MAIN RESULTS}

The main aim of this paper is to show that piecewise linear maps of type $2^{\infty}$ do not exist. The basic scheme behind the proof is as follows. First we realize that any piecewise monotone map (in particular any piecewise linear map) of type $2^{\infty}$ has an infinite $\omega$-limit set. But it is also known that any map of type $2^{\infty}$ having an infinite $\omega$-limit set also has a doubling period solenoid. Then we show that a piecewise linear map cannot have any doubling period solenoids.

We start with some notations and definitions.

Let $I$ be a real compact interval and $C(I)$ be the set of continuous maps from $I$ into itself. Let $\mathbb{N}$ be the set of positive integers. A point $p \in I$ is a periodic point of a map $f \in C(I)$ if $f^{n}(p)=p$ for some $n \in \mathbb{N}$. The period of $p$ is the least such integer $n$, and the orbit of $p$ under $f$ is the set $\operatorname{orb}_{f}(p)=\left\{f^{k}(p): k=0,1, \ldots, n-1\right\}$. We refer to such an orbit as to a periodic orbit of $f$ of period $n$. A periodic point of period 1 is called a fixed point. Similarly, we say that a sequence $\left(I_{k}\right)_{k=0}^{n-1}$ of closed subintervals of $I$ is periodic of period $n$ if they have disjoint interiors and $f\left(I_{k}\right) \subset I_{k+1}$ for any $k=0,1, \ldots, n-2$ and $f\left(I_{n-1}\right) \subset I_{0}$. In this case we call $C=\bigcup_{k=0}^{n-1} I_{k}$ a cycle of periodic intervals. Further, we say that $A \subset I$ is a solenoid of $f$ (the term solenoid is used, e.g., in [MSt2]) if there are a strictly increasing sequence $\left(k_{n}\right)_{n=1}^{\infty}$ of positive integers and periodic sequences $\left(I_{k}^{n}\right)_{k=0}^{k_{n}-1}$ of closed intervals of period $k_{n}$ such that $\bigcup_{k=0}^{k_{n}-1} I_{k}^{n} \supset \bigcup_{k=0}^{k_{n+1}-1} I_{k}^{n+1}$ for any $n$ and $A=\bigcap_{n=1}^{\infty} \bigcup_{k=0}^{k_{n}-1} I_{k}^{n}$. We call the family $\left\{\left(I_{k}^{n}\right)_{k=0}^{k_{n}-1}\right\}_{n=1}^{\infty}$ a covering of $A$ of type

Received by the editors October 10, 1994.

1991 Mathematics Subject Classification. Primary 58F08; Secondary 26A18, 54H20.

Key words and phrases. Asymptotically periodic point, piecewise linear map, piecewise monotone map, solenoid, wandering interval, $\omega$-limit set.

A part of the work on this paper was done during the stay of the second author at the University of Murcia. The invitation and the support of this institution is gratefully acknowledged.

This work has been partially supported by the DGICYT grant numbers PB91-0575 and PB941159 and by the Slovak grant agency, grant number 1/1470/1994.

The main result of this paper was announced at the "Thirty years after Sharkovskii's Theorem. New perspectives" Conference, held in La Manga (Murcia), Spain, June 13-17th, 1994.

The authors are greatly indebted to the referee for many helpful suggestions which enabled them to shorten and simplify the paper.

(C) 1997 American Mathematical Society 
$\left(k_{n}\right)_{n=1}^{\infty}$. If a solenoid admits a covering of type $\left(2^{n}\right)_{n=1}^{\infty}$ we call it a doubling period solenoid of $f$.

The set of all limit points of the trajectory $\left(f^{n}(x)\right)_{n=0}^{\infty}$ of a point $x$ is called the $\omega$-limit set of $x$ under $f$ and denoted by $\omega_{f}(x)$. We say that $x$ is an asymptotically periodic point of $f$ if for some periodic point $p,\left|f^{n}(x)-f^{n}(p)\right| \rightarrow 0$ when $n \rightarrow \infty$. We also describe this situation by saying that $x$ is attracted by (the orbit of ) $p$.

The key to understanding the dynamics of maps from $C(I)$ is the well known Sharkovskii theorem [Sh1]. Consider the Sharkovskii ordering of the set $\mathbb{N} \cup\left\{2^{\infty}\right\}$ :

$$
\begin{aligned}
3 & \succ 5 \succ 7 \succ \cdots \succ 2 \cdot 3 \succ 2 \cdot 5 \succ 2 \cdot 7 \succ \cdots \succ 4 \cdot 3 \succ 4 \cdot 5 \succ 4 \cdot 7 \succ \cdots \succ \cdots \\
& \succ 2^{n} \cdot 3 \succ 2^{n} \cdot 5 \succ 2^{n} \cdot 7 \succ \cdots \succ \cdots \succ 2^{\infty} \succ \cdots \succ 2^{n} \succ \cdots \succ 4 \succ 2 \succ 1 .
\end{aligned}
$$

We will also use the symbol $\succeq$ in the natural way. For $n \in \mathbb{N} \cup\left\{2^{\infty}\right\}$ we denote by $S(n)$ the set $\{k \in \mathbb{N}: n \succeq k\}\left(S\left(2^{\infty}\right)\right.$ stands for the set $\left.\left\{1,2,4, \ldots, 2^{k}, \ldots\right\}\right)$. Let $f \in C(I)$ and $\operatorname{Per}(f)$ be the set of periods of its periodic points. The Sharkovskii theorem says that there exists $n \in \mathbb{N} \cup\left\{2^{\infty}\right\}$ such that $\operatorname{Per}(f)=S(n)$. The converse of the Sharkovskii theorem also holds, that is, for every $n \in \mathbb{N} \cup\left\{2^{\infty}\right\}$ there exists $f \in C(I)$ with $\operatorname{Per}(f)=S(n)$ (see [Sh1], [Sh2]). If $\operatorname{Per}(f)=S(n)$, then $f$ is said to be of type $n$. So any map $f \in C(I)$ is of some type and for every $n \in \mathbb{N} \cup\left\{2^{\infty}\right\}$ there is a map of type $n$. When speaking of types we consider them to be ordered by the Sharkovskii ordering. So if a map $f$ is of type $2^{\infty}$ or greater than $2^{\infty}$, then $\operatorname{Per}(f)=\left\{1,2, \ldots, 2^{k}, \ldots\right\}$ or $f$ has a periodic point with period not a power of 2 , respectively.

It turns out that the Sharkovskii theorem allows us to organize a classification of the maps from $C(I)$ in terms of their dynamical complexity. So, maps of type $2^{n}, n=0,1,2, \ldots$, are simple: all their points are asymptotically periodic (see [Le], [Co], cf. [Sh2]). On the other hand, maps of type greater than $2^{\infty}$ have a very complicated dynamics. For instance, they are chaotic in the sense of $\mathrm{Li}$ and Yorke [LY], which means that there exists an uncountable set $S \subset I$ containing no asymptotically periodic points (which implies that $\omega_{f}(x)$ is infinite for each $x \in S$, see $[\mathrm{Ba}])$ and such that

$$
\limsup _{n \rightarrow \infty}\left|f^{n}(x)-f^{n}(y)\right|>0, \quad \liminf _{n \rightarrow \infty}\left|f^{n}(x)-f^{n}(y)\right|=0
$$

for any $x, y \in S, x \neq y$. Maps of type $2^{\infty}$ are located somewhere between these two groups. Indeed, there are examples of maps $F, G$ of type $2^{\infty}$ respectively having only asymptotically periodic points $[\mathrm{BP}]$ and chaotic in the sense of $\mathrm{Li}$ and Yorke $[\mathrm{Sm}]$.

Let $\mathbb{R}$ be the real line and $J \subset \mathbb{R}$ a (not necessarily compact) interval. We say that a continuous map $f: J \rightarrow \mathbb{R}$ is piecewise monotone (resp. piecewise linear) if there are points $\inf I=a_{0}<a_{1}<\cdots<a_{n}=\sup I$ such that for every $k \in\{1,2, \ldots, n\}$, the restriction of $f$ to the interval $\left(a_{k-1}, a_{k}\right)$ is (not necessarily strictly) monotone (resp. linear and non-constant). Note that, according to the definitions, piecewise monotone maps can have constant pieces, while piecewise linear maps cannot. In the case of piecewise linear maps, notice also that the consecutive linear pieces need not be alternatively increasing and decreasing.

One could wonder whether the converse of the Sharkovskii theorem holds for piecewise monotone (or even polynomial) maps from $C(I)$. It is well known that the answer is affirmative. For instance, the classical logistic family $\left\{F_{\lambda}\right\}_{\lambda \in[0,4]}$ defined by $F_{\lambda}(x)=\lambda x(1-x)$ contains examples of maps of all types in the Sharkovskii 
ordering (see [Gu1]). On the other hand, compare this with the family of "tent" maps $\left\{G_{\mu}\right\}_{\mu \in[0,1]}$ defined by $G_{\mu}(x)=\mu(1-|2 x-1|)$. The map $G_{\mu}$ is of type greater than $2^{\infty}$ if $\mu>1 / 2$ but $G_{\mu}$ is of type 1 for any $\mu \leqslant 1 / 2$ (see e.g. [BP]). So, a question remains: does the converse of the Sharkovskii theorem hold for piecewise linear maps? Examples of piecewise linear maps of all types except of type $2^{\infty}$ are well known (see e.g. Corollary 2.2.9 in $[\mathrm{ALM}]$ ) and the map $F$ from $[\mathrm{BP}]$ of type $2^{\infty}$ mentioned earlier consists of an infinite number of non-constant linear pieces. Further, the map $H \in C([0,1])$ defined by $H(x)=\min \left\{\kappa, G_{1}(x)\right\}, \kappa \approx 0.8249 \ldots$ is of type $2^{\infty}$ (see [MS]) but it has a constant piece and so it is not piecewise linear. No examples of piecewise linear maps of type $2^{\infty}$ are known. The main result of this paper says that such examples do not exist.

We will use the following proposition which is a part of folklore knowledge.

Proposition. If $f \in C(I)$ is a piecewise monotone map of type $2^{\infty}$, then it has a doubling period solenoid.

To see that the Proposition holds true recall a well known result implicit in several of Sharkovskii's papers and proved in [Sm] stating that every infinite $\omega$ limit set of a map of type $2^{\infty}$ is contained in a doubling period solenoid. So it is sufficient to show that any piecewise monotone map of type $2^{\infty}$ has an infinite $\omega$ limit set. But this is proved in [BC], Proposition II.28 and Proposition VI.10. (The proof is based on the ideas similar to those from [FSh] and though the definition of piecewise monotonicity used in $[\mathrm{BC}]$ is more restrictive than the one used in the present paper, one can see that the proof remains valid also for maps which are piecewise monotone in our sense.)

The Proposition seems to be useful for applications. For instance, we have strongly used it in [JS] to show that it is always possible to find, for any given map of type $2^{\infty}$ (not necessarily piecewise monotone), maps of type $2^{k}, k<\infty$, as close to it as required. On the other hand, it is not easy to follow its proof in the sense that the proofs of Propositions II.28 and VI.10 from [BC] involve several auxiliary results, some of them not being very well known. Therefore we will give below a short elementary proof.

The following theorem is the key result of the present paper.

Theorem. If $f \in C(I)$ is piecewise linear, then it has no doubling period solenoids.

After the first version of this paper was submitted, we learned of a recent paper by Martens and Tresser [MT] where this result was strengthened (see Section 3 below). The Proposition and Theorem give

Corollary. There are no piecewise linear maps of type $2^{\infty}$ in $C(I)$.

Remark. The Sharkovskii theorem also holds for the set $C(\mathbb{R})$ of continuous maps from the real line into itself (in this case we have the additional possibility $\operatorname{Per}(f)=$ $\emptyset)$. It turns out that $C(\mathbb{R})$ does not contain any piecewise linear maps of type $2^{\infty}$ either. To prove it, assume $f \in C(\mathbb{R})$ is a piecewise linear map of type $2^{\infty}$. Take a homeomorphism $\phi:(0,1) \rightarrow \mathbb{R}$ and construct $g=\phi^{-1} \circ f \circ \phi$. We can extend $g$ to a piecewise monotone map $g_{0} \in C([0,1])$ of type $2^{\infty}$. By the Proposition, $g_{0}$ has a doubling period solenoid $A$. Let $\left\{\left(I_{k}^{n}\right)_{k=0}^{2^{n}-1}\right\}_{n=1}^{\infty}$ be a covering of $A$ of type $\left(2^{n}\right)_{n=1}^{\infty}$. Since $g_{0}(\{0,1\}) \subset\{0,1\}$ (note that both $\phi$ at the points $0^{+}, 1^{-}$and $f$ at the points $-\infty,+\infty$ have infinite limits) and $A$ cannot contain any asymptotically periodic points, $\bigcup_{k=0}^{2^{l}-1} I_{k}^{l} \subset(0,1)$ for some $l$ large enough. Since the restriction of 
$g^{2^{l}}$ to the interval $I_{0}^{l}$ is of type $2^{\infty}$, so is the restriction of $f^{2^{l}}$ to the interval $\phi\left(I_{0}^{l}\right)$. This contradicts our Theorem.

\section{Proofs}

Before going to the proofs fix some notations. The convex hull of a set $A \subset I$ will be denoted by conv $A$, $\operatorname{int} A$ is the interior of $A$. If $f: A \rightarrow B$ and $C \subset A$, $\left.f\right|_{C}$ is the restriction of $f$ to $C$. A subinterval $J$ of $I$ containing no asymptotically periodic points of $f \in C(I)$ and such that $f^{n}(J) \cap f^{k}(J)=\emptyset$ for any $n>k \geqslant 0$ will be called a wandering interval of $f$.

Let $f \in C(I)$ be piecewise monotone. In what follows we will say that $t \in I$ is a turning point of $f$ if there are points $u<t<v$ in $I$ such that the maps $\left.f\right|_{[u, t]}$ and $\left.f\right|_{[t, v]}$ are non-constant and one of them is increasing while the other is decreasing (the monotonicity may not be strict). Each connected component of the set of turning points of $f$ will be called a turning interval of $f$. Notice that a turning interval may degenerate to a point. Clearly, $f$ has a finite number of turning intervals, and if $T$ is a turning interval of $f$, then $\left.f\right|_{T}$ is constant. One can also check that if $T$ is a turning interval of $f^{n}$ for some $n$, then there exists $0 \leqslant k<n$ such that $f^{k}(T)$ intersects some turning interval of $f$.

Proof of Proposition. Assume the contrary. Then all turning points of $f$ are asymptotically periodic. It is not restrictive to assume that all turning points of $f$ are attracted by fixed points (take into account the properties of turning intervals described above to replace $f$ if necessary by an appropriate $f^{2^{k}}$ ).

Let $P$ be a periodic orbit of $f$ of period four and denote conv $P=[a, b]$. Since the set $\{a, b\}$ cannot be mapped by $f$ onto itself, one of the points where the map $\left.f\right|_{[a, b]}$ attains its absolute extremum (with no loss of generality we can suppose that it is the minimum) must belong to $(a, b)$. This point (denote it by $c$ ) is a turning point of $f$. Let $d<c<e$ be the closest points to $c$ with the property $f(d)=f(e)=a$. Clearly, the interval $[d, e]$ contains no fixed points of $f$. Denote the fixed point attracting $c$ by $p$. Let for example $p<d$. Then there is an $m$ large enough so that $f^{m}(c)<d$ and $f^{m}(d)=f^{m}(e)=b$. In particular, $[d, e] \subset f^{m}([d, c]) \cap f^{m}([c, e])$ $\left(f^{m}\right.$ has a horseshoe). But it is well known and easy to prove that then the type of $f$ is greater than $2^{\infty}$.

We will need the following lemma.

Lemma. Let $J$ be an interval and $f: J \rightarrow \mathbb{R}$ be a piecewise linear map with at most one point for which $f^{\prime}$ does not exist. Let $m \in \mathbb{N}$ and $q_{0}<t_{1}<q_{1}<t_{2}<q_{2}<\cdots<$ $t_{m}<q_{m}$ be points from $J$ for which $f^{\prime}$ exists. Then there is an $r \in\{0,1, \ldots, m\}$ such that

$$
\prod_{i=0}^{m} f^{\prime}\left(q_{i}\right)=f^{\prime}\left(q_{r}\right) \cdot \prod_{i=1}^{m} f^{\prime}\left(t_{i}\right) .
$$

Proof. If $f$ is linear with slope $\rho$, then for an arbitrary choice of $r$ each of the sides equals $\rho^{m+1}$. Now assume that $c$ is the only point where $f^{\prime}$ does not exist. If $c<q_{0}$ or $c>q_{m}$, then the lemma holds for the same reason as above. So let $q_{i}<c<t_{i+1}$ for some $i \in\{0,1, \ldots, m-1\}$ or $t_{j}<c<q_{j}$ for some $j \in$ $\{1,2, \ldots, m\}$. In the former case we have $f^{\prime}\left(q_{0}\right)=f^{\prime}\left(t_{1}\right), \ldots, f^{\prime}\left(q_{i-1}\right)=f^{\prime}\left(t_{i}\right)$ and $f^{\prime}\left(t_{i+1}\right)=f^{\prime}\left(q_{i+1}\right), \ldots, f^{\prime}\left(t_{m}\right)=f^{\prime}\left(q_{m}\right)$, so it suffices to take $r=i$. Similarly, in the latter case take $r=j$. 
Proof of Theorem. Assume the contrary. Let $f$ be a piecewise linear map having a doubling period solenoid $A$. Let $\left\{\left(I_{k}^{n}\right)_{k=0}^{2^{n}-1}\right\}_{n=1}^{\infty}$ be a covering of $A$ of type $\left(2^{n}\right)_{n=1}^{\infty}$. For any $n$, write $C^{n}=\bigcup_{k=0}^{2^{n}-1} I_{k}^{n}$. We are going to show that without loss of generality we may assume that

$$
\text { the intervals } I_{k}^{n}, k=0,1, \ldots, 2^{n}-1 \text {, are pairwise disjoint for any } n \geqslant 1 \text {. }
$$

To this end denote $J_{k}^{n}=\operatorname{conv}\left(A \cap I_{k}^{n}\right)$. Since $A$ cannot contain any asymptotically periodic points, the intervals $J_{k}^{n}, k=0,1, \ldots, 2^{n}-1$, are pairwise disjoint. There is an $m$ large enough such that if $c$ is a relative extremum of $f$ belonging to $C^{m}$, then it also belongs to $A$. Since $f(A) \subset A$ this implies that $f\left(J_{k}^{m}\right) \subset J_{k+1}^{m}$ for any $k=0,1, \ldots, 2^{m}-2$ and $f\left(J_{2^{m}-1}^{m}\right) \subset J_{0}^{m}$. Consequently, it is not restrictive to assume (1) (replacing if necessary $f$ by $\left.f^{2^{i}}\right|_{J_{0}^{i}}$ for $i=m-1$ if $m>1$ ).

Further observe that

$$
\text { the length of the intervals } I_{k}^{n} \text { converges uniformly to zero when } n \rightarrow \infty \text {. }
$$

Otherwise, there exist $\epsilon>0$ and $k_{n}$ for any $n$ such that $I_{k_{n}}^{n}$ has length greater than $\epsilon$. Let $a_{n}$ be the midpoint of each interval $I_{k_{n}}^{n}$ and $\left(a_{n_{i}}\right)_{i}$ be a subsequence of $\left(a_{n}\right)_{n}$ converging to some $a$. It is not restrictive to suppose that $\left|a_{n_{i}}-a\right|<\frac{\epsilon}{3}$ for any $i$. Then $I_{k_{n_{i}}}^{n_{i}} \cap I_{k_{n_{i+1}}}^{n_{i+1}} \neq \emptyset$ and so $I_{k_{n_{i+1}}}^{n_{i+1}} \subset I_{k_{n_{i}}}^{n_{i}}$ for any $i$ (see (1). This means that there is an interval $J$ such that $J \subset A$. Obviously, $f^{j}(J) \cap f^{m}(J)=\emptyset$ for any $j>m \geqslant 0$. Since $A$ does not contain any asymptotically periodic points, $J$ is a wandering interval of $f$ which is impossible since by [MMS] piecewise linear maps have no wandering intervals.

Denote by $C$ the set of points for which $f^{\prime}$ does not exist (in general this set is larger than the set of relative extrema of $f$ ). From (2) and the fact that $C$ is finite it follows that there is an $l$ such that

(3) $C \cap C^{l} \subset A$ and each $I_{k}^{n}$ contains at most one point from $C$ for any $n \geqslant l$.

Fix each $I_{0}^{n}$ in such a way that $I_{0}^{n+1} \subset I_{0}^{n}$ for any $n$. Then $I_{k}^{n+1}, I_{k+2^{n}}^{n+1} \subset I_{k}^{n}$ for any $n$ and any $k=0,1, \ldots, 2^{n}-1$. Let $K_{k}^{n}$ be the set consisting of all points lying between $\operatorname{int} I_{k}^{n+1}$ and $\operatorname{int} I_{k+2^{n}}^{n+1}$. According to (1), $K_{k}^{n}$ is a non-degenerate closed interval. Since for a fixed $n$ the intervals $K_{k}^{n}$ are pairwise disjoint and $f\left(K_{k}^{n}\right) \supset K_{k+1}^{n}$ for any $k=0,1, \ldots, 2^{n}-2$ and $f\left(K_{2^{n}-1}^{n}\right) \supset K_{0}^{n}$, there exists a periodic orbit $P_{n}$ of period $2^{n}$ containing exactly one point (which will be denoted by $p_{k}^{n}$ ) from each $K_{k}^{n}$.

For any $n \geqslant l$ define $\delta_{n}=\left(f^{2^{n}}\right)^{\prime}\left(p_{0}^{n}\right)=f^{\prime}\left(p_{0}^{n}\right) \cdot f^{\prime}\left(p_{1}^{n}\right) \cdots f^{\prime}\left(p_{2^{n}-1}^{n}\right)$. Notice that $\delta_{n}$ is well defined, since by (3) the set $C \cap C^{n} \subset A$ and so it cannot contain any periodic points. We claim that

$$
\left|\delta_{n}\right|>1 \text { for any } n \geqslant l \text {. }
$$

Indeed let $i \geqslant l$ be such that $\left|\delta_{i}\right| \leqslant 1$ and define the map $g=\left.f^{2^{i}}\right|_{I_{0}^{i}} \in C\left(I_{0}^{i}\right)$. Consider the maximal interval containing $p_{0}^{i}$ on which $g^{\prime}$ exists. Since $g$ is piecewise linear and $p_{0}^{i}$ is a fixed point of $g$, there exists an endpoint $c$ of this interval such that $g^{j}(c) \rightarrow p_{0}^{i}$ when $j \rightarrow \infty$ if $\left|\delta_{i}\right|<1$ and $g^{2}(c)=c$ if $\left|\delta_{i}\right|=1$. In either case $c$ is an asymptotically periodic point of $g$ and then an asymptotically periodic point of $f$. Clearly we can choose $c$ with the additional property of not being an endpoint of the interval $I_{0}^{i}$, and then $g^{\prime}(c)$ does not exist. This means that $f^{m}(c) \in C$ 


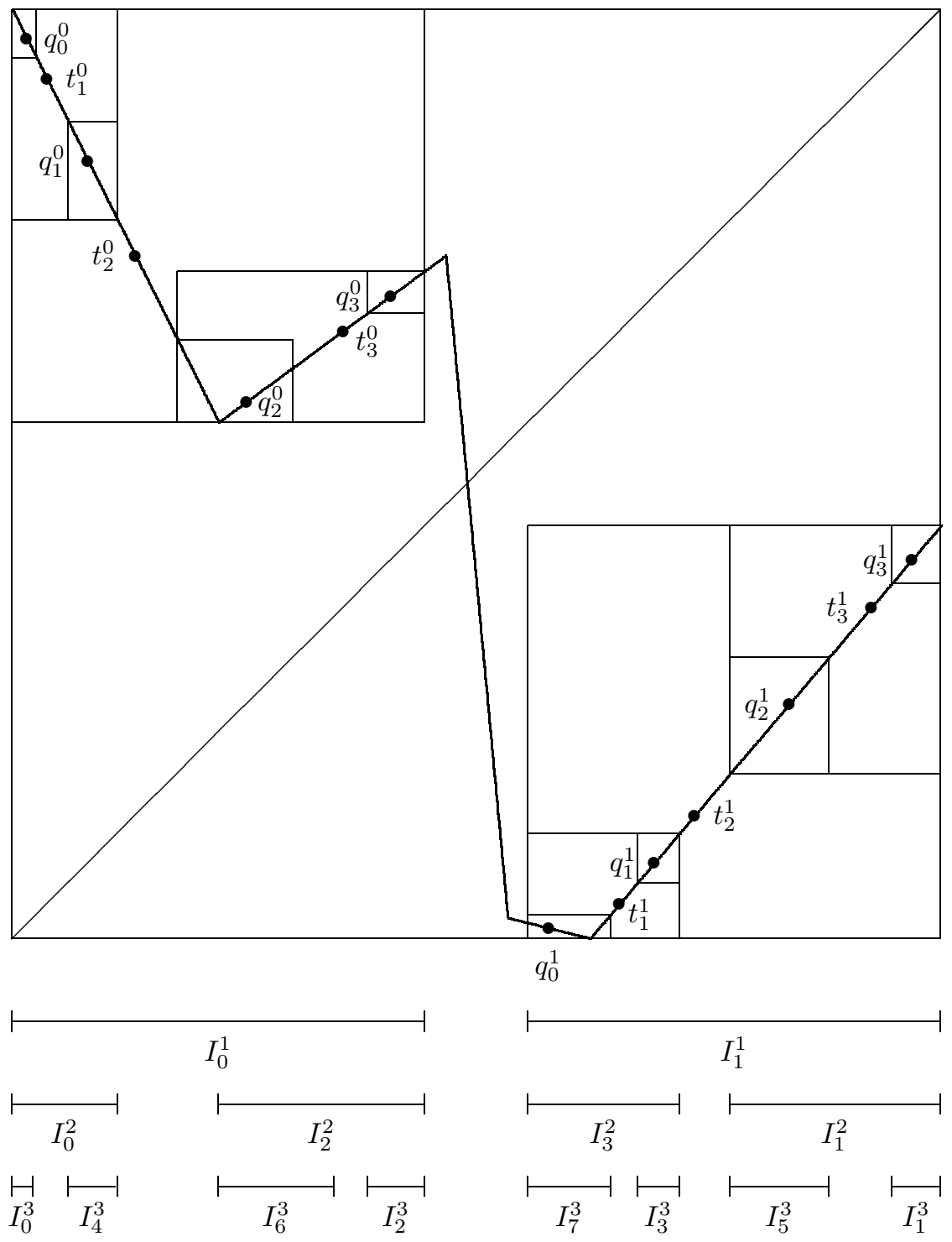

Figure 1. The structure of the map $f$ from the proof of Theorem. Here we assume $l=1, n=3$.

for some $m \in\left\{0,1, \ldots, 2^{i}-1\right\}$, which is impossible because $f^{m}(c)$ would be an asymptotically periodic point of $f$ belonging to $C \cap C^{i}$ and then, by (3), to $A$.

Let $L$ be the (finite) set of all possible values of $f^{\prime}$ (where it is defined) and construct $M=\left\{\rho_{0} \cdot \rho_{1} \cdots \rho_{2^{l}-1}: \rho_{k} \in L, k=0,1, \ldots, 2^{l}-1\right\}$, also a finite set. Fix 
$n>l$ and let $x \in I_{0}^{n}$ be such that $\delta=\left(f^{2^{n}}\right)^{\prime}(x)$ exists. We are going to show that

$$
\delta=\delta_{l} \cdot \delta_{l+1} \cdots \delta_{n-1} \cdot \nu \quad \text { for some } \nu \in M .
$$

Define $Q=\left\{f^{j}(x)\right\}_{j=0}^{2^{n}-1}, T=\bigcup_{s=l}^{n-1} P_{s}$ and write also $Q_{k}=Q \cap I_{k}^{l}, T_{k}=T \cap I_{k}^{l}$, $k=0,1, \ldots, 2^{l}-1$. Recall that $f^{j}(x) \in I_{j}^{n}$ for any $j=0,1, \ldots, 2^{n}-1$ and that each point $p_{r}^{s}$ of the periodic orbit $P_{s}$ lies between the intervals $I_{r}^{s+1}$ and $I_{r+2^{s}}^{s+1}$. Then the cardinality of the sets $Q_{k}$ and $T_{k}$ is respectively $2^{n-l}$ and $2^{n-l}-1$. Moreover, if $q_{0}^{k}<q_{1}^{k}<q_{2}^{k}<\cdots<q_{2^{n-l}-1}^{k}$ and $t_{1}^{k}<t_{2}^{k}<\cdots<t_{2^{n-l}-1}^{k}$ are the elements of $Q_{k}$ and $T_{k}$, we have $q_{0}^{k}<t_{1}^{k}<q_{1}^{k}<t_{2}^{k}<q_{2}^{k}<\cdots<t_{2^{n-l}-1}^{k}<q_{2^{n-l}-1}^{k}$ (see Figure 1). Also, recall that by (3) each interval $I_{k}^{l}$ can contain at most one point from $C$. Therefore, by the Lemma, for every $k \in\left\{0,1, \ldots, 2^{l}-1\right\}$ there exists a point $\widetilde{q_{k}} \in Q_{k}$ such that

$$
\prod_{q \in Q_{k}} f^{\prime}(q)=f^{\prime}\left(\widetilde{q_{k}}\right) \cdot \prod_{t \in T_{k}} f^{\prime}(t)
$$

Hence

$$
\delta=\left(f^{2^{n}}\right)^{\prime}(x)=\prod_{q \in Q} f^{\prime}(q)=\prod_{k=0}^{2^{l}-1} \prod_{q \in Q_{k}} f^{\prime}(q)=\prod_{k=0}^{2^{l}-1}\left(f^{\prime}\left(\widetilde{q_{k}}\right) \cdot \prod_{t \in T_{k}} f^{\prime}(t)\right) .
$$

To prove (5) realize that the number $\prod_{k=0}^{2^{l}-1} f^{\prime}\left(\widetilde{q_{k}}\right)$ belongs to $M$ and

$$
\prod_{k=0}^{2^{l}-1} \prod_{t \in T_{k}} f^{\prime}(t)=\prod_{s=l}^{n-1} \prod_{t \in P_{s}} f^{\prime}(t)=\prod_{s=l}^{n-1} \delta_{s}
$$

In particular (note that $p_{0}^{n} \in I_{0}^{n}$ ), for any $n>l$ there exists $\nu_{n} \in M$ such that

$$
\delta_{n}=\delta_{l} \cdot \delta_{l+1} \cdots \delta_{n-1} \cdot \nu_{n} .
$$

We claim that there exists a number $\delta^{\prime}>1$ such that

$$
\left|\delta_{n}\right| \geqslant \delta^{\prime} \quad \text { for any } n>l .
$$

Indeed, notice that the set $\left\{\nu_{n}\right\}_{n=l+1}^{\infty}$ is finite and take $m>l$ such that $\left\{\nu_{n}\right\}_{n=l+1}^{\infty}=$ $\left\{\nu_{l+1}, \nu_{l+2}, \ldots, \nu_{m}\right\}$. By (4) we have $\left|\delta_{n}\right|>1$ for $n=l+1, l+2, \ldots, m$. Now take any $n>m$. Then there is $w \in\{l+1, l+2, \ldots, m\}$ with $\nu_{n}=\nu_{w}$ and so $\left|\delta_{n}\right|=\left|\delta_{l} \cdot \delta_{l+1} \cdots \delta_{m-1} \cdot \delta_{m} \cdots \delta_{n-1} \cdot \nu_{n}\right|=\left|\delta_{l} \cdot \delta_{l+1} \cdots \delta_{m-1} \cdot \delta_{m} \cdots \delta_{n-1} \cdot \nu_{w}\right|=$ $\left|\delta_{w}\right| \cdot\left|\delta_{w} \cdots \delta_{n-1}\right|>\left|\delta_{w}\right|$ (we have used (4) and (6)). Thus it is sufficient to take $\delta^{\prime}=\min \left\{\left|\delta_{l+1}\right|,\left|\delta_{l+2}\right|, \ldots,\left|\delta_{m}\right|\right\}$.

Clearly, there exists some integer $r$ not depending on $n$ such that the number of points for which $\left(\left.f^{2^{n}}\right|_{I_{0}^{n}}\right)^{\prime}$ does not exist is less than $r$. On the other hand, if $n$ is large enough we have from (5) and (7) that $\left|\left(f^{2^{n}} \mid I_{0}^{n}\right)^{\prime}(x)\right|>r$ in each point $x$ for which $\left(\left.f^{2^{n}}\right|_{I_{0}^{n}}\right)^{\prime}(x)$ exists. This is impossible, since in the opposite case the length of the interval $f^{2^{n}}\left(I_{0}^{n}\right)$ would be greater than the length of $I_{0}^{n}$.

\section{Final Remarks and open Questions}

We are going to discuss possible generalizations of our results as well as some related conjectures.

First we recall the role of wandering intervals in the dynamics and introduce a class of maps without wandering intervals which could seem at a first glance to be 
a candidate for the generalization of our result on the non-existence of piecewise linear maps of type $2^{\infty}$.

The dynamics of maps from $C(I)$ can be substantially complicated due to the presence of wandering intervals. For example, if a map of type $2^{\infty}$ is chaotic in the sense of $\mathrm{Li}$ and Yorke, then it must have wandering intervals (see [Sm]), while for each piecewise strictly monotone map (i.e., piecewise monotone and constant in no subinterval of $I$ ) having no wandering intervals there is a residual set $R \subset$ $I$ such that for any $x \in R$ its $\omega$-limit set is either a periodic orbit, a cycle of periodic intervals or a solenoid (this follows for example from Theorem 2.4 and Propositions $2.8,2.9$ and 4.2 in $[\mathrm{Pr}])$.

Fortunately, for a large class of continuous maps wandering intervals do not exist. Recall that a point $c$ is said to be a critical point of $f$ if $f$ is differentiable in $c$ and $f^{\prime}(c)=0$. If additionally $f$ is of class $C^{k+1}$ in a neighborhood of $c$ and $f^{(k)}(c) \neq 0$ for some $k \geq 2$, then $c$ is called non-flat. Let $A(I)$ denote the class of all maps $f$ from $C(I)$ with the following properties:

1. There are points $\min I=a_{0}<a_{1}<\cdots<a_{n}=\max I$ such that $\left.f\right|_{\left[a_{k-1}, a_{k}\right]}$ is $C^{1}$ for any $k=1,2, \ldots, n$ (in the points $a_{k-1}$ and $a_{k}$ we mean one-sided derivatives). Further, each $\left(\left.f\right|_{\left[a_{k-1}, a_{k}\right]}\right)^{\prime}$ is a bounded variation map which does not vanish in $\left(a_{k-1}, a_{k}\right)$.

2. For any $k=0,1,2, \ldots, n$, either $a_{k}$ is a non-flat critical point of $f$, or the one-sided derivatives of $f$ in $a_{k}$ do not vanish.

Notice that $A(I)$ contains all (non-constant) analytic maps and all piecewise linear maps. In a remarkable paper by Martens, de Melo and van Strien [MMS] (cf. [MSt2], pp. 267-268), which generalizes earlier results by Denjoy [De], Guckenheimer [Gu2], de Melo and van Strien [MSt1], Lyubich [Ly1] and Blokh and Lyubich [BL], it was proved that if $f \in A(I)$, then it has no wandering intervals (in fact they prove their result for a more general class of maps).

One would expect that our result on the nonexistence of maps of type $2^{\infty}$ in the class of piecewise linear maps could be extended to larger classes of maps, in particular to the class $A_{0}(I)$ of maps from $A(I)$ whose derivatives never vanish. More precisely, $f \in C(I)$ is said to belong to $A_{0}(I)$ if there are points $\min I=$ $a_{0}<a_{1}<\cdots<a_{n}=\max I$ such that $\left.f\right|_{\left[a_{k-1}, a_{k}\right]}$ is $C^{1}$ for any $k=1,2, \ldots, n$ (in the points $a_{k-1}$ and $a_{k}$ we mean one-sided derivatives) and each $\left(\left.f\right|_{\left[a_{k-1}, a_{k}\right]}\right)^{\prime}$ is a bounded variation map which does not vanish in $\left[a_{k-1}, a_{k}\right]$. However this is not the case. We give below an example of a map $f \in A_{0}([0,1])$ of type $2^{\infty}$ which is in fact piecewise $C^{\infty}$. The map $f$ has a doubling period solenoid (see the Proposition or the construction of $f$ below). This is basically the only possible type of a solenoid of $f$. More precisely, it follows from the construction of $f$ (cf. also [AJS]) that if $\left(k_{n}\right)_{n=1}^{\infty}$ is the type of a covering of a solenoid of $f$, then each $k_{n}$ is a power of two.

We are going to define $f$. First notice that for any $a>0$ it is easy to construct a strictly increasing $C^{\infty}$ map $\Psi_{a}:[0,1] \rightarrow[0, a]$ such that $\Psi_{a}(0)=0, \Psi_{a}(1)=a$, $\Psi_{a}^{\prime}(0)=\Psi_{a}^{\prime}(1)=1$ and $\Psi_{a}^{(n)}(0)=\Psi_{a}^{(n)}(1)=0, n=2,3, \ldots$ (here $f^{(n)}$ denotes the $n$-th derivative of $f$ ). Moreover, the maps $\Psi_{a}$ can be defined in such a way that for any $\epsilon>0$ and $n \in \mathbb{N}$ there exists $\delta_{\epsilon, n}>0$ such that if $|a-1|<\delta_{\epsilon, n}$, then $\left|\Psi_{a}^{\prime}(x)-1\right|<\epsilon$ and $\left|\Psi_{a}^{(k)}(x)\right|<\epsilon$ for any $x \in[0,1]$ and $k=2,3, \ldots, n$. Now construct a sequence $\left(\gamma_{n}\right)_{n=0}^{\infty}$ of positive real numbers with $\gamma_{0}=1$ and $\frac{\gamma_{n}}{\gamma_{n-1}}<\frac{1}{3}$ for any $n \geqslant 1$. Put $\kappa_{n}=\frac{\gamma_{n-1}-\gamma_{n}-\gamma_{n+1}}{\gamma_{n-1}-2 \gamma_{n}}, \epsilon_{n}=\frac{1}{n}\left(\frac{\gamma_{n-1}}{3}\right)^{n-1}$ for any $n \geqslant 1$. Modifying if 


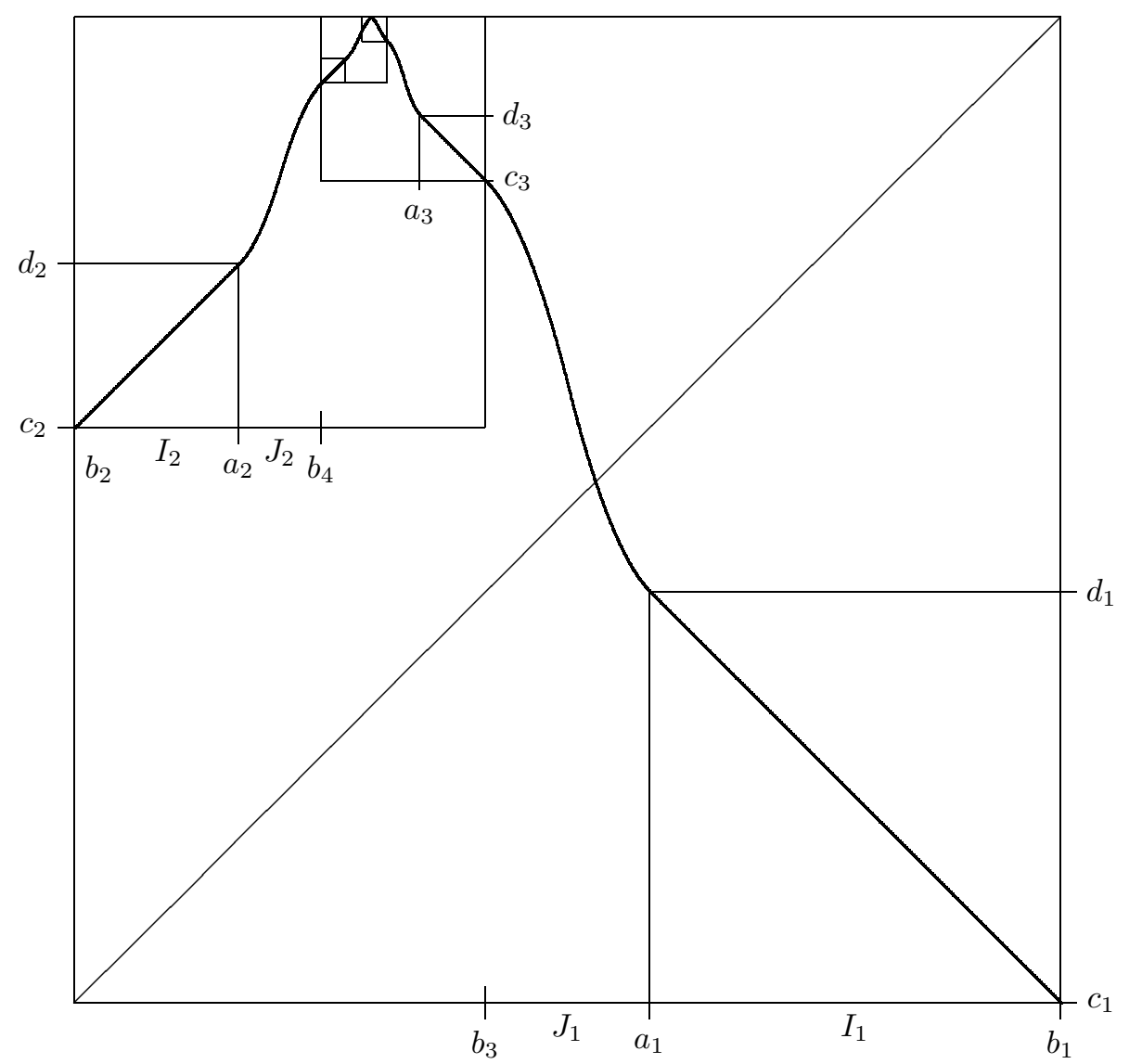

Figure 2. The graph of the map $f$ from the example of Section 3. We have distorted the values of numbers $\gamma_{n}$ to increase the clarity of the picture.

necessary $\left(\gamma_{n}\right)_{n=0}^{\infty}$ we can assume that $\left|\kappa_{n}-1\right|<\delta_{\epsilon_{n}, n}$ for any $n$. So, $\left|\Psi_{\kappa_{n}}^{\prime}(x)-1\right|<$ $\epsilon_{n}$ and $\left|\Psi_{\kappa_{n}}^{(k)}(x)\right|<\epsilon_{n}$ for any $x \in[0,1]$ and $k=2,3, \ldots, n$. Further, define $b_{1}=1$ and $b_{n}=b_{n-1}+(-1)^{n-1} \gamma_{n-2}, n=2,3, \ldots$, and put $a_{n}=b_{n}+(-1)^{n} \gamma_{n}$. Write also $c_{n}=1-\gamma_{n-1}, d_{n}=1-\gamma_{n-1}+\gamma_{n}$ for any $n \geqslant 1$ and put $I_{n}=\operatorname{conv}\left\{a_{n}, b_{n}\right\}$, $J_{n}=\operatorname{conv}\left\{b_{n+2}, a_{n}\right\}$. Observe that $c_{n+2}-d_{n}=\gamma_{n-1}-\gamma_{n}-\gamma_{n+1}$ and $\left|b_{n+2}-a_{n}\right|=$ $\gamma_{n-1}-2 \gamma_{n}$ for any $n$ and so $\kappa_{n}=\frac{c_{n+2}-d_{n}}{\left|b_{n+2}-a_{n}\right|}$. Finally, put $c=\sum_{n=1}^{\infty}(-1)^{n-1} \gamma_{n}$.

We are ready to define $f$. For any $n \geq 1$ and $x \in I_{n}$ (resp. $x \in J_{n}$ ), put $f(x)=c_{n}+\left|x-b_{n}\right|$ (resp. $f(x)=d_{n}+\left|b_{n+2}-a_{n}\right| \Psi_{\kappa_{n}}\left(\left|\frac{x-a_{n}}{b_{n+2}-a_{n}}\right|\right)$ ). Finally put $f(c)=1$ (see Figure 2).

It is easy to check that $f$ is well defined, $f \in C([0,1])$ and it is of type $2^{\infty}$. Moreover, $\left.f\right|_{[0, c)}$ and $\left.f\right|_{(c, 1]}$ are clearly $C^{\infty}$ maps. Further, recall that $\left|b_{n+2}-a_{n}\right|=$ $\gamma_{n-1}-2 \gamma_{n}>\frac{\gamma_{n-1}}{3}$ and then $\left|f^{(k)}(x)\right|<\frac{1}{n}$ for any $x \in I_{n} \cup J_{n}$ and $k=2,3, \ldots, n$, while $\left|f^{\prime}(x)-1\right|<\frac{1}{n}$ for any $x \in I_{n} \cup J_{n}$ if $n$ is even and $\left|f^{\prime}(x)+1\right|<\frac{1}{n}$ for any $x \in I_{n} \cup J_{n}$ if $n$ is odd. Therefore, $\lim _{x \rightarrow c} f^{(n)}(x)=0$ for any $n \geqslant 2$ while 
$\lim _{x \rightarrow c^{-}} f^{\prime}(x)=1$ and $\lim _{x \rightarrow c^{+}} f^{\prime}(x)=-1$. Using the L'Hôpital rule we get that $\left.f\right|_{[0, c]}$ and $\left.f\right|_{[c, 1]}$ are $C^{\infty}$ maps (whose first derivatives do not vanish).

So $A_{0}(I)$ failed as a candidate for the extension of our result. Still, some degree of generalization for it must be possible. Denote by $K_{2}(I)$ the class of continuous maps $I \rightarrow I$ consisting of two pieces with zero Schwarzian derivative. So, $f \in K_{2}(I)$ if $f \in C(I)$ and for some $c \in \operatorname{int} I, f(x)=\frac{a_{1} x+b_{1}}{c_{1} x+d_{1}}$ for any $x \leqslant c$ and $f(x)=\frac{a_{2} x+b_{2}}{c_{2} x+d_{2}}$ for any $x \geqslant c\left(a_{i} d_{i}-b_{i} c_{i} \neq 0, i=1,2\right)$. Let $K(I)$ be the class of similar maps with an arbitrary (finite) number of pieces of monotonicity. In $[\mathrm{Ko}]$ it is proved that if $f \in K_{2}(I)$, then it has no doubling period solenoid and so, by the Proposition, is not of type $2^{\infty}$. In [AJS] it is proved that maps from $K_{2}(I)$ have no solenoids at all. We conjecture that the same result remains valid for maps from $K(I)$. Recently Martens and Tresser [MT] extended our Theorem by showing that piecewise linear maps do not have any solenoids at all. Thus, they answered in the affirmative the above conjecture for the subclass of $K(I)$ consisting of all piecewise linear maps.

It is easy to check that for the map $f$ we discussed in the example above there is a sequence $\left(p_{n}\right)_{n=1}^{\infty}$ with every $p_{n}$ a periodic point of $f$ of period $2^{n}$ such that $\left|\left(f^{2^{n}}\right)^{\prime}\left(p_{n}\right)\right| \rightarrow 1$ when $n \rightarrow \infty$. On the other hand, in [MMS] (cf. also [MSt2], p. 268) it is proved that if $f \in B(I)$ (where $B(I)$ is the class of $C^{2}$ maps having only non-flat critical points), there are a real number $a_{f}>1$ and an integer $n_{f}$ depending only on $f$ such that if $p$ is a periodic point of $f$ of period $n>n_{f}$, $\left|f^{(n)}(p)\right|>a_{f}$. Our example shows that this result cannot be generalized to maps from $A_{0}(I)$. However, we conjecture that it holds for maps from $K(I)$.

In connection with the result mentioned at the beginning of this section it was conjectured for some years that if $f$ is "reasonably smooth" (ideally if $f \in A(I)$ ), then for almost every $x \in I, \omega_{f}(x)$ is either a periodic orbit, a cycle of periodic intervals or a solenoid. Indeed, this result has been proved in particular for the family of logistic maps [Ly2], but unfortunately it does not hold even for polynomial maps (see [BKNS]). We conjecture that if $f \in K(I)$, then $\omega_{f}(x)$ is either a periodic orbit or a cycle of periodic intervals for almost every $x$.

It is hard to propose more general settings where the above described properties may hold. In particular, it is not clear whether the existence of piecewise analytic maps (with non-vanishing derivatives) of type $2^{\infty}$ should be reasonably expected or not.

\section{REFERENCES}

[AJS] L. Alsedà, V. Jiménez López and L. Snoha, On 1-difactors of Markov graphs and the prevalence of doubling period solenoids, preprint (1995).

[ALM] L. Alsedà, J. Llibre and M. Misiurewicz, Combinatorial Dynamics and Entropy in Dimension One, World Scientific Publ., Singapore, 1993. MR 95j:58042

[Ba] B. Barna, Über die Iteration reeller Functionen II, Publ. Math. Debrecen 13 (1966), 169-172. MR 34:4419

[BC] L. S. Block and W. A. Coppel, Dynamics in One Dimension, Lecture Notes in Math., vol. 1513, Springer, Berlin, 1992. MR 93g:58091

[BKNS] H. Bruin, G. Keller, T. Nowicki and S. van Strien, Wild Cantor attractors exist, Ann. of Math. 143 (1996), 97-130. CMP 96:07

[BL] A. M. Blokh and M. Yu. Lyubich, Non-existence of wandering intervals and structure of topological attractors of one dimensional dynamical systems 2. The smooth case, Ergod. Th. \& Dynam. Sys. 9 (1989), 751-758. MR 91e:58101

[BP] G. J. Butler and G. Pianigiani, Periodic points and chaotic functions in the unit interval, Bull. Austral. Math. Soc. 18 (1978), 255-265. MR 58:13203 
[Co] W. A. Coppel, The solutions of equations by iteration, Proc. Camb. Phil. Soc. 51 (1955), 41-43. MR 16:577a

[De] A. Denjoy, Sur les courbes définies par les équations differentielles à la surface du tore, Jour. Math. Pures Appl. 11 (1932), 333-375.

[FSh] V. V. Fedorenko and A. N. Sharkovskii, Continuous mappings of an interval with closed sets of periodic point, An investigation of differential and differential-difference equations, Inst. Math. Ukrain. Acad. Sci., Kiev, 1980, pp. (137-145) (Russian). MR 83i: 58083

[Gu1] J. Guckenheimer, On the bifurcations of maps of the interval, Invent. Math. 39 (1977), 165-178. MR 55:11312

[Gu2] J. Guckenheimer, Sensitive dependence on initial conditions for one dimensional maps, Comm. Math. Phys. 70 (1979), 133-160. MR 82c:58037

[JS] V. Jiménez López and L. Snoha, All maps of type $2^{\infty}$ are boundary maps, Proc. Amer. Math. Soc. (to appear).

[Ko] S. F. Kolyada, Interval maps with zero Schwarzian; in Functional-differential Equations and Their Applications, Inst. Math. Ukrain. Acad. Sci., Kiev, 1985, pp. (47-57) (Russian). MR 88i:58080

[Le] Z. L. Leibenzon, Investigation of some properties of a continuous pointwise mapping of an interval onto itself, having an application in the theory of nonlinear oscillations, Prikl. Mat. i Mekh. 17 (1953), 351-360 (Russian). MR 14:1072e

[Ly1] M. Yu. Lyubich, Non-existence of wandering intervals and structure of topological attractors of one dimensional dynamical systems 1. The case of negative Schwarzian derivative, Ergod. Th. \& Dynam. Sys. 9 (1989), 737-750. MR 91e:58100

[Ly2] M. Yu. Lyubich, Combinatorics, geometry and attractors of quasi-quadratic maps, Ann. of Math. 140 (1994), 347-404. MR 95j:58108

[LY] T. Y. Li and J. A. Yorke, Period three implies chaos, Amer. Math. Monthly 82 (1975), 985-992. MR 52:5898

[MMS] M. Martens, W. de Melo and S. van Strien, Julia-Fatou-Sullivan theory for real onedimensional dynamics, Acta Math. 168 (1992), 271-318. MR 93d:58137

[MS] M. Misiurewicz and J. Smítal, Smooth chaotic functions with zero topological entropy, Ergod. Th. \& Dynam. Sys. 8 (1988), 421-424. MR 90a:58118

[MSt1] W. de Melo and S. van Strien, A structure theorem in one-dimensional dynamics, Ann. of Math. 129 (1989), 519-546. MR 90m:58106

[MSt2] W. de Melo and S. van Strien, One-Dimensional Dynamics, Springer, Berlin, 1993. MR 95a:58035

[MT] M. Martens and C. Tresser, Forcing of periodic orbits for interval maps and renormalization of piecewise affine maps, Proc. Amer. Math. Soc. 124 (1996), 2863-2870. MR 96k: 58180

[Pr] C. Preston, Iterates of piecewise monotone mappings of an interval, Lecture Notes in Math. 1347, Springer, Berlin, 1988. MR 89m:58109

[Sh1] A. N. Sharkovskii, Coexistence of cycles of a continuous mapping of the line into itself, Ukrain. Math. Zh. 16 (1964), 61-71 (Russian). MR 96j:58058

[Sh2] A. N. Sharkovskii, On cycles and the structure of a continuous mapping, Ukrain. Math. Zh. 17 (1965), 104-111 (Russian).

[Sm] J. Smítal, Chaotic functions with zero topological entropy, Trans. Amer. Math. Soc. 297 (1986), 269-282. MR 87m:58107

Departamento de Matemáticas, Universidad de Murcia, Campus de Espinardo, Aptdo. de Correos 4021, 30100 Murcia, Spain

E-mail address: vjimenez@fcu.um.es

Department of Mathematics, Faculty of Natural Sciences, Matej Bel University, Tajovského 40, 97401 Banská Bystrica, Slovakia

E-mail address: snoha@bb.sanet.sk 